\title{
MAGIC observations of the diffuse $\gamma$-ray emission in the vicinity of the Galactic center ${ }^{\star}$
}

MAGIC Collaboration: V. A. Acciari ${ }^{1,2}$, S. Ansoldi ${ }^{3,24}$, L. A. Antonelli ${ }^{4}$, A. Arbet Engels ${ }^{5}$, D. Baack ${ }^{6}$, A. Babić ${ }^{7}$, B. Banerjee ${ }^{8}$, U. Barres de Almeida ${ }^{9}$, J. A. Barrio ${ }^{10}$, J. Becerra González ${ }^{1,2}$, W. Bednarek ${ }^{11}$, L. Bellizzi ${ }^{12}$, E. Bernardini ${ }^{13,17}$, A. Berti ${ }^{14}$, J. Besenrieder ${ }^{15}$, W. Bhattacharyya ${ }^{13}$, C. Bigongiari ${ }^{4}$, A. Biland ${ }^{5}$, O. Blanch ${ }^{16}$, G. Bonnoli ${ }^{12}$, Ž. Bošnjak ${ }^{7}$, G. Busetto ${ }^{17}$, R. Carosi ${ }^{18}$, G. Ceribella $^{15}$, Y. Chai ${ }^{15}$, A. Chilingaryan ${ }^{19}$, S. Cikota ${ }^{7}$, S. M. Colak ${ }^{16}$, U. Colin ${ }^{15}$, E. Colombo ${ }^{1,2}$, J. L. Contreras ${ }^{10}$, J. Cortina ${ }^{20}$, S. Covino ${ }^{4}$, V. D'Elia ${ }^{4}$, P. Da Vela ${ }^{18}$, F. Dazzi ${ }^{4}$, A. De Angelis ${ }^{17}$, B. De Lotto ${ }^{3}$, M. Delfino ${ }^{16,27}$, J. Delgado ${ }^{16,27}$, D. Depaoli ${ }^{14}$, F. Di Pierro ${ }^{14}$, L. Di Venere ${ }^{14}$, E. Do Souto Espiñeira ${ }^{16}$, D. Dominis Prester $^{7}$, A. Donini ${ }^{3}$, D. Dorner ${ }^{21}$, M. Doro ${ }^{17}$, D. Elsaesser ${ }^{6}$, V. Fallah Ramazani ${ }^{22}$, A. Fattorini ${ }^{6}$, A. Fernández-Barral ${ }^{17}$, G. Ferrara ${ }^{4}$, D. Fidalgo ${ }^{10}$, L. Foffano ${ }^{17}$, M. V. Fonseca ${ }^{10}$, L. Font ${ }^{23}$, C. Fruck ${ }^{15, \star \star}$, S. Fukami ${ }^{24}$, R. J. García López ${ }^{1,2}$, M. Garczarczyk ${ }^{13}$, S. Gasparyan ${ }^{19}$, M. Gaug ${ }^{23}$, N. Giglietto ${ }^{14}$, F. Giordano ${ }^{14}$, N. Godinović ${ }^{7}$, D. Green ${ }^{15}$, D. Guberman ${ }^{16}$, D. Hadasch ${ }^{24}$, A. Hahn ${ }^{15}$, J. Herrera ${ }^{1,2}$, J. Hoang ${ }^{10}$, D. Hrupec ${ }^{7}$, M. Hütten ${ }^{15}$, T. Inada ${ }^{24}$, S. Inoue ${ }^{24}$, K. Ishio ${ }^{15}$, Y. Iwamura ${ }^{24, \star \star}$, L. Jouvin ${ }^{16}$, D. Kerszberg ${ }^{16}$, H. Kubo ${ }^{24}$, J. Kushida ${ }^{24}$, A. Lamastra ${ }^{4}$, D. Lelas ${ }^{7}$, F. Leone ${ }^{4}$, E. Lindfors ${ }^{22}$, S. Lombardi ${ }^{4}$, F. Longo ${ }^{3,28}$, M. López ${ }^{10}$, R. López-Coto ${ }^{17}$, A. López-Oramas ${ }^{1,2}$, S. Loporchio ${ }^{14}$, B. Machado de Oliveira Fraga ${ }^{9}$, C. Maggio ${ }^{23}$, P. Majumdar ${ }^{8}$, M. Makariev ${ }^{25}$, M. Mallamaci ${ }^{17}$, G. Maneva ${ }^{25}$, M. Manganaro ${ }^{7}$, K. Mannheim ${ }^{21}$, L. Maraschi ${ }^{4}$, M. Mariotti ${ }^{17}$, M. Martínez ${ }^{16}$, S. Masuda ${ }^{24}$, D. Mazin ${ }^{15,24}$, S. Mićanović ${ }^{7}$, D. Miceli ${ }^{3}$, M. Minev ${ }^{25}$, J. M. Miranda ${ }^{12}$, R. Mirzoyan ${ }^{15}$, E. Molina ${ }^{26}$, A. Moralejo ${ }^{16}$, D. Morcuende ${ }^{10}$, V. Moreno ${ }^{23}$, E. Moretti $^{16}$, P. Munar-Adrover ${ }^{23}$, V. Neustroev ${ }^{22}$, C. Nigro $^{13}$, K. Nilsson ${ }^{22}$, D. Ninci ${ }^{16}$, K. Nishijima ${ }^{24}$, K. Noda ${ }^{24}$, L. Nogués ${ }^{16}$, M. Nöthe ${ }^{6}$, S. Nozaki ${ }^{24}$, S. Paiano ${ }^{17}$, J. Palacio ${ }^{16}$, M. Palatiello ${ }^{3}$, D. Paneque ${ }^{15}$, R. Paoletti ${ }^{12}$, J. M. Paredes ${ }^{26}$, P. Peñil ${ }^{10}$, M. Peresano ${ }^{3}$, M. Persic ${ }^{3,29}$, P. G. Prada Moroni ${ }^{18}$, E. Prandini ${ }^{17}$, I. Puljak ${ }^{7}$, W. Rhode ${ }^{6}$, M. Ribó ${ }^{26}$, J. Rico ${ }^{16}$, C. Righi ${ }^{4}$, A. Rugliancich ${ }^{18}$, L. Saha ${ }^{10}$, N. Sahakyan ${ }^{19}$, T. Saito ${ }^{24}$, S. Sakurai ${ }^{24}$, K. Satalecka ${ }^{13}$, K. Schmidt ${ }^{6}$, T. Schweizer ${ }^{15}$, J. Sitarek ${ }^{11}$, I. Šnidarić ${ }^{7}$, D. Sobczynska ${ }^{11}$, A. Somero ${ }^{1,2}$, A. Stamerra ${ }^{4}$, D. Strom ${ }^{15}$, M. Strzys ${ }^{15,24, \star \star}$, Y. Suda ${ }^{15}$, T. Surić ${ }^{7}$, M. Takahashi ${ }^{24}$, F. Tavecchio ${ }^{4}$, P. Temnikov ${ }^{25}$, T. Terzić ${ }^{7}$, M. Teshima ${ }^{15,24}$,

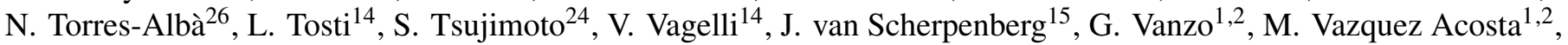
C. F. Vigorito ${ }^{14}$, V. Vitale ${ }^{14}$, I. Vovk ${ }^{15,24, \star \star}$, M. Will ${ }^{15}$, and D. Zarić ${ }^{7}$

(Affiliations can be found after the references)

Received 11 October 2019 / Accepted 24 May 2020

\section{ABSTRACT}

Aims. In the presence of a sufficient amount of target material, $\gamma$-rays can be used as a tracer in the search for sources of Galactic cosmic rays (CRs). Here we present deep observations of the Galactic center (GC) region with the MAGIC telescopes and use them to infer the underlying CR distribution and to study the alleged $\mathrm{PeV}$ proton accelerator at the center of our Galaxy.

Methods. We used data from $\approx 100 \mathrm{~h}$ observations of the GC region conducted with the MAGIC telescopes over five years (from 2012 to 2017). Those were collected at high zenith angles (58-70 deg), leading to a larger energy threshold, but also an increased effective collection area compared to low zenith observations. Using recently developed software tools, we derived the instrument response and background models required for extracting the diffuse emission in the region. We used existing measurements of the gas distribution in the GC region to derive the underlying distribution of CRs. We present a discussion of the associated biases and limitations of such an approach.

Results. We obtain a significant detection for all four model components used to fit our data (Sgr A*, "Arc", G0.9+0.1, and an extended component for the Galactic Ridge). We observe no significant difference between the $\gamma$-ray spectra of the immediate GC surroundings, which we model as a point source $\left(\mathrm{Sgr} \mathrm{A}^{*}\right)$ and the Galactic Ridge. The latter can be described as a power-law with index 2 and an exponential cut-off at around $20 \mathrm{TeV}$ with the significance of the cut-off being only $2 \sigma$. The derived cosmic-ray profile hints to a peak at the GC position and with a measured profile index of $1.2 \pm 0.3$ is consistent with the $1 / r$ radial distance scaling law, which supports the hypothesis of a CR accelerator at the GC. We argue that the measurements of this profile are presently limited by our knowledge of the gas distribution in the GC vicinity.

Key words. gamma rays: general - gamma rays: ISM - Galaxy: center - cosmic rays

\footnotetext{
$\star$ Tables and sky maps are only available at the CDS via anonymous ftp to cdsarc.u-strasbg.fr (130.79.128.5) or via http://cdsarc.u-strasbg.fr/viz-bin/cat/J/A+A/642/A190

$\star \star$ Corresponding authors: Christian Fruck, Ievgen Vovk, Yuki Iwamura and Marcel Strzys (e-mail: contact.magic@mpp.mpg.de).
} 


\section{Introduction}

The Galactic center (GC) is one of the most extraordinary regions in the very high energy (VHE, $>100 \mathrm{GeV}) \gamma$-ray sky, containing a rich variety of sources capable of accelerating charged particles and thereby producing VHE $\gamma$-ray emission (van Eldik 2015; Aharonian et al. 2006a; H.E.S.S. Collaboration 2006). If those $\gamma$-rays are produced in hadronic interactions, their sources may also contribute to the overall cosmic-ray "sea" filling the region with energized charged particles. This sea of cosmic rays (CRs) is believed to be responsible for the diffuse Galactic plane emission detected by EGRET (Hunter et al. 1997) and later studied by Fermi/LAT (Abdo et al. 2010), as well as the central $\gamma$-ray ridge detected with all major Imaging Atmospheric Cherenkov Telescopes (IACTs, H.E.S.S. Collaboration 2006; Archer et al. 2016; Ahnen et al. 2017a). The detected $\gamma$ rays originate primarily from deep inelastic nuclear interactions of the high-energetic particles with the surrounding gas, which can also be traced via its emission in the radio band (CS emission, Tsuboi et al. 1999; CO emission, Oka et al. 1998). Thus, deep $\gamma$-ray observations in the $\mathrm{TeV}$ energy range can be used to infer the underlying CR density, provided that the gas distribution is reliably measured.

This idea has been applied in earlier studies using observations of the central $\approx \pm 1^{\circ}$ region (in latitude) of the Galaxy (corresponding to $\approx 300 \mathrm{pc}$ at a distance of $8.5 \mathrm{kpc}$ ) with the High Energy Stereoscopic System (H.E.S.S.), suggesting an inhomogeneous distribution of CRs around the GC (Aharonian et al. 2006b; H.E.S.S. Collaboration 2016, 2018). Furthermore, those data indicate that the central super-massive black hole of our Galaxy may itself accelerate particles to $\mathrm{PeV}\left(10^{15} \mathrm{eV}\right)$ energies.

Observing the GC region with the MAGIC telescopes is only possible at large zenith distances $\left(>58^{\circ}\right)$, implying an increased optical thickness of the atmosphere and larger distance to the shower maximum, which leads to a stronger dilution of the Cherenkov light. On the other hand, for geometric reasons, these data benefit from an increased collection area $\left(\approx 1 \mathrm{~km}^{2}\right.$, Ahnen et al. 2017a) and, thus, a boost of sensitivity at energies above several TeVs.

Ahnen et al. (2017a) already presented the first part of the MAGIC GC observation campaign in 2012-2015, consisting of $\approx 70 \mathrm{~h}$ of exposure. Apart from the detection of multi-TeV emission from the Galactic plane, those data suggested the presence of a new VHE source close to the so-called radio "Arc" in the GC vicinity, detected also by other telescopes (Archer et al. 2016; H.E.S.S. Collaboration 2018). We continued observing the GC during 2015-2017, adding up to a total exposure time of $\approx 100 \mathrm{~h}$. In addition, we employ the recently developed 2D likelihood analysis package SkyPrism (Vovk et al. 2018), which provides a more sensitive analysis of extended sources for MAGIC.

This paper is organized as follows. In Sect. 2, we describe the MAGIC observations, the data selection strategy, and the data analysis techniques. In Sect. 3, we provide a detailed description of the various analysis steps and report on their results. In Sect. 4, we estimate the biases, stemming from our background modeling and analysis approach. Finally, Sects. 5 and 6 provide a general discussion and a summary of the obtained results.

\section{MAGIC observations of the Galactic center region}

The Major Atmospheric Gamma Imaging Cherenkov (MAGIC) telescopes are two $17 \mathrm{~m}$ diameter IACTs, located at a distance of $85 \mathrm{~m}$ from each other at an altitude of $2200 \mathrm{~m}$ a.s.l. at the Roque de los Muchachos Observatory on the Canary Island of La Palma, Spain $\left(28^{\circ} \mathrm{N}, 18^{\circ} \mathrm{W}\right)$.
The telescopes record flashes of Cherenkov light produced by extensive air showers (EAS) initiated in the upper atmosphere by $\gamma$-ray photons within a field of view of $1.5^{\circ}$ (in radius). Both telescopes are operated together in a coincidence trigger stereoscopic mode, in which only events that simultaneously trigger both telescopes are recorded and analyzed (Aleksić et al. 2016). For low-zenith distance observations and for $E>220 \mathrm{GeV}$, the integral sensitivity of MAGIC is $(0.66 \pm 0.03) \%$ in units of the Crab Nebula flux (C.U.) for $50 \mathrm{~h}$ of observation (Aleksić et al. 2016). At larger zenith angles (above $60^{\circ}$ ), at which the GC is observable at La Palma, the MAGIC collection area reaches $1 \mathrm{~km}^{2}$ at the energies above $10 \mathrm{TeV}$ (Ahnen et al. 2017a).

MAGIC observations of the GC region which we used for this study were carried out between April 2012 and May 2017 (typically from March to July). The observation time after quality selection cuts is $\approx 100 \mathrm{~h}$. The data were taken in the so-called "Wobble" mode (Fomin et al. 1994), centered on Sgr A* with a pointing offset of $0.4^{\circ}$.

The acquired data were processed with the standard MAGIC Analysis and Reconstruction Software (MARS, Zanin et al. 2013). The steps involved include the selection of good data quality periods, low-level processing of camera images, calculation of image parameters, and the reconstruction of the energy and incoming direction of the primary $\gamma$-rays. The last step is performed using the machine learning technique Random Forests (Albert et al. 2008), where Monte Carlo events and real background data are used for training. This technique is also used for background suppression through event classification.

At the quality selection step, we removed events corresponding to known temporary hardware issues or recorded during bad weather periods. As the full data sample contains some nights with weak moonlight, we also excluded time periods with sky brightness $\gtrsim 2.5$ times the typical dark night sky brightness. Data recorded with higher sky brightness lead to biases when analyzed with the same pipeline that is used for data that has been recorded during dark nights but can, in principle, still be used when accepting a higher energy threshold when applying special analysis settings (see Ahnen et al. 2017b). In order to limit our selection to only good quality data, we applied cuts on several instrument and weather related parameters. These include the mean photomultiplier currents, the event trigger rate, the number of stars detected by the MAGIC star-guider cameras, and the cloud or aerosol induced absorption in the telescope's field of view. The latter is derived from measurements with an infra-red pyrometer and a LIDAR system (Gaug et al. 2014; Fruck et al. 2014).

\section{Data analysis}

A high-level analysis of the acquired data was performed with the set of utilities available in the MAGIC SkyPrism package (Vovk et al. 2018). SkyPrism provides a set of routines, aimed at 2D spatial likelihood analysis of MAGIC data, which allows us to self-consistently account for sources of complex morphology within the field of view. Furthermore, it also incorporates algorithms for computing the instrument point spread function, exposure and background maps, which we intensively use throughout the analysis presented here.

\subsection{Diffuse background model construction}

The wobble observational scheme (Fomin et al. 1994) we used here provides a simultaneous background estimate from comparison of several adjunct (albeit exposed to slightly different 
sky regions) positions. Still, the applicability of this approach is limited by the distance between the wobble pointings and the target, which in our case is $d_{w}=0.4^{\circ}$. In practice, a source with an extension comparable to or larger than twice the offset distance $\left(0.8^{\circ}\right)$, in the same direction, will still (partially) contribute to the background measured in the same camera region. The diffuse emission of the Galactic plane clearly exceeds this limit, so a special treatment is necessary.

Such contamination of the background map with excess $\gamma$ ray signal can be avoided if contributions from locations close to known $\gamma$-ray sources are excluded during map construction (Vovk et al. 2018). We thus mask out camera regions that correspond to the Galactic plane and use the rest of the camera (free from known or expected sources) to estimate the corresponding background. This approach, implemented in the SkyPrism package, allows us to significantly reduce the background bias.

Due to the remaining limitations of our background modeling technique and the wobble scheme we used, we cannot completely remove the possible bias in the background estimation from a source as extended as the diffuse Galactic plane emission. Our estimates, described in detail in Sect. 4.1 and illustrated in Fig. 7 , demonstrate that in the $l=\left[-1.5^{\circ} ; 1.5^{\circ}\right]$ longitude range along the plane, the remaining bias is within $10-20 \%$ of the assumed local source luminosity throughout the Galactic plane and does not exceed $30-50 \%$ (equivalent to $\approx 3 \%$ of background) in the outskirts. At the same time, the flux bias is rather constant across the plane and its variations, averaged in the latitude range $b=\left[-0.2^{\circ} ; 0.2^{\circ}\right]$ do not exceed $\approx 1 \%$ of the background, which corresponds to $10-20 \%$ of the estimated source flux for a highly extended source.

\subsection{MAGIC view of the Galactic center region}

The large $\left(>58^{\circ}\right)$ zenith angle GC observations imply an increased energy threshold of $\approx 1 \mathrm{TeV}$, although an analysis is also possible at even lower energies given that the studied source is bright enough (Ahnen et al. 2017a). Thus, we performed the spectral analysis in the $400 \mathrm{GeV}-50 \mathrm{TeV}$ energy range; the morphology study of the diffuse emission in the GC region was done above $\gamma$-ray energies of $1 \mathrm{TeV}$.

The sky map (above $E=1 \mathrm{TeV}$ ) of the GC vicinity, produced with the described diffuse background estimation scheme, is shown in Fig. 1. The Galactic plane is visible over $2^{\circ}$ across the image. The significance of this detection, computed using the CS radio emission profile (Tsuboi et al. 1999) as an approximation (see Sects. 3.3 and 3.4 for details of the method), results in $\mathrm{a} \approx 17$ standard deviations $(\sigma)$ incompatibility with the null hypothesis of background and point sources only. Other sources visible in the image are Sgr A*, G0.9+0.1 and the so-called "Arc", detected with significances of $\approx 48 \sigma, \approx 11 \sigma$ and $\approx 6.4 \sigma$, respectively, while also propagating uncertainties of the background and exposure model.

\subsection{Galactic plane brightness scan}

The CR distribution profile in the GC surroundings should roughly correspond to the brightness distribution of the detected $\gamma$-ray emission. Previous measurements have already shown evidence that the $\gtrsim 100 \mathrm{GeV}$ brightness of the Galactic plane is peaking towards the Sgr $\mathrm{A}^{*}$, indicating a concentration of cosmic-rays (H.E.S.S. Collaboration 2016). The presence of an extended central component, reported by the H.E.S.S. Collaboration (2018), also speaks in favour of this assumption.

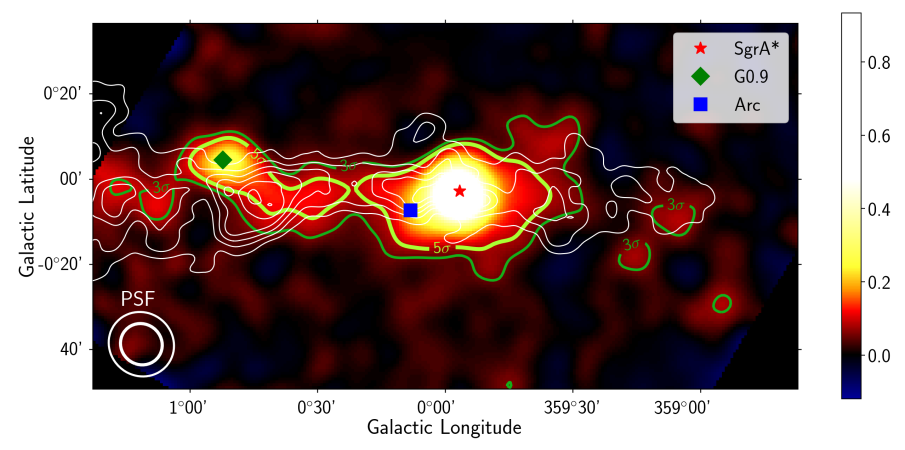

Fig. 1. Sky map (excess in units of background) of the GC region in Galactic coordinates at energies above $1 \mathrm{TeV}$, smeared with a kernel resembling the MAGIC PSF. The pre-trial statistical significance of regions with excess counts is highlighted by green contours at the levels $5 \sigma$ and $3 \sigma$. The (smeared) MAGIC PSF is indicated by $39 \%$ and $68 \%$ containment contours. The white contours show radio line emission from CS molecules, tracing dense gas (Tsuboi et al. 1999).

The cosmic-ray distribution around the GC can be inferred by solving the integral equation

$S(x, y)=A \int \rho_{\mathrm{gas}}(x, y, z) \rho_{\mathrm{CR}}(x, y, z) \mathrm{d} z$

where $S(x, y)$ is the image plane $\gamma$-ray brightness distribution, $\rho_{\text {gas }}$ and $\rho_{\mathrm{CR}}$ are the number densities of the gas and CRs, respectively, and $A$ is a factor, which takes into account the proton-proton interaction cross-section, distance to observer, and additional constants. A proper solution of this equation requires knowledge of the full 3D gas density distribution $\rho_{\text {gas }}(x, y, z)$, which is challenging to obtain. Indeed, while in the image plane $(x, y)$ the resolution of radio surveys reaches $\approx 0.01^{\circ}$ (for instance the CS $J=1-0$ emission radio survey of Tsuboi et al. 1999) equivalent to $1-2 \mathrm{pc}$ scale, the line-of-sight distance $z$ can hardly be obtained with an accuracy better than several tens of parsecs. For this reason we solve an approximate expression of Eq. (1):

$S(x, y) \approx A \int \rho_{\text {gas }} \mathrm{d} z \times \int \rho_{\mathrm{CR}} \mathrm{d} z=P_{\text {gas }}(x, y) P_{\mathrm{CR}}(x, y)$,

which splits the problem into the projected gas (directly inferred from the radio data) and cosmic-ray distributions $P_{\text {gas }}(x, y)$ and $P_{\mathrm{CR}}(x, y)$ respectively. To avoid degeneracy, we just consider radially symmetric cosmic-ray profiles $\rho_{\mathrm{CR}} \propto r^{-\alpha}$ (with $r$ being the distance from the GC) and their projections onto the image plane. These simplifications result in a certain bias of our measurement, which we quantify in Sect. 4.2.

First, we test whether a homogeneous cosmic-ray distribution $\rho_{\mathrm{CR}}=$ const is consistent with the MAGIC data. For this, we produced an excess event profile of the $b=\left[-0.2^{\circ} ; 0.2^{\circ}\right]$ stripe, centered at the GC position, and the corresponding MAGIC exposure profile using the features of SkyPrism. The resulting, exposure-normalized, Galactic plane profile above $1.2 \mathrm{TeV}$ is shown in Fig. 2. Fitted with a simple model, containing the Sgr A* and G0.9 point sources, the "Arc" source, and the diffuse emission model $S(x, y)$ (computed from the CS emission maps with $\rho_{\mathrm{CR}}=$ const $)$, it results in $\chi^{2} /$ d.o.f. $\approx 69 / 46$ degrees of freedom, equivalent to $\approx 2.4 \sigma$ disagreement of the data with the model.

In order to investigate if the MAGIC data are in a better agreement with a $\rho_{\mathrm{CR}} \neq$ const type cosmic-ray profiles, we used a grid search for the optimal value of $\alpha$ using the profile shown in Fig. 2 and we estimated the cosmic-ray density 


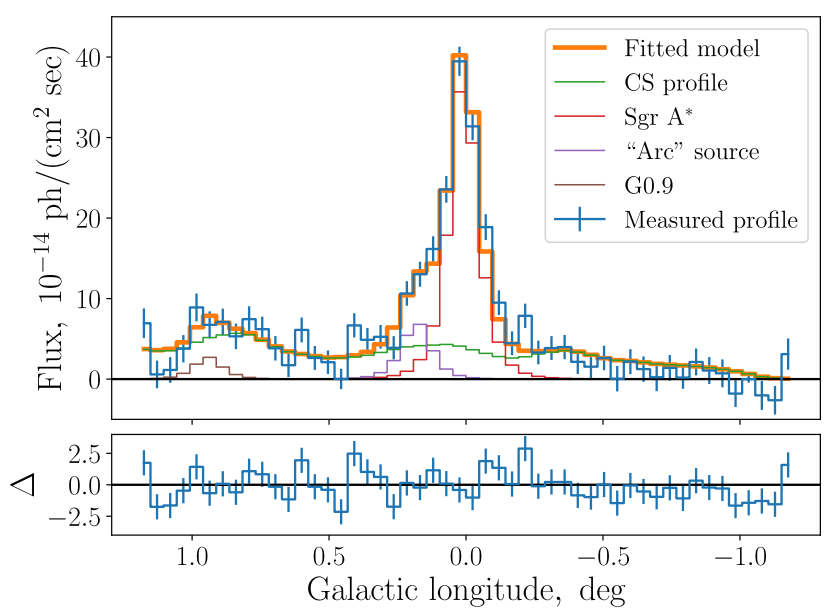

Fig. 2. Top: brightness scan of the $b=\left[-0.2^{\circ} ; 0.2^{\circ}\right]$ stripe of the Galactic plane in the energy range above $1.2 \mathrm{TeV}$. Blue entries denote the MAGIC measurements, whereas the orange line is the best-fit model to them, composed of the CS profile, Sgr A* point source and the extended "Arc". Bottom: residuals of the fit in the units of measurement uncertainties.

$P_{\mathrm{CR}}(d)=S(d) / P_{\mathrm{gas}}(d)$ as function of the projected off-center distance $d$ using a full maximum likelihood fit of the measured $\gamma$-ray brightness around the GC.

The results of the likelihood-profile scan are shown in Fig. 3, where the density $\rho_{\mathrm{CR}}$ is converted to the cosmic-ray energy density $w_{\mathrm{CR}}$ using the same procedure as in H.E.S.S. Collaboration (2016) (formula (2) of the methods section):

$w_{\mathrm{CR}}\left(\geq 10 E_{\gamma}\right) \approx 1.8 \times 10^{-2}\left(\frac{\eta_{N}}{1.5}\right)^{-1}\left(\frac{L_{\gamma}\left(\geq E_{\gamma}\right)}{10^{34} \mathrm{erg} \mathrm{s}^{-1}}\right)\left(\frac{M}{10^{6} M_{\odot}}\right)^{-1}$,

where $w_{\mathrm{CR}}$ is in $\mathrm{eV} \mathrm{cm}^{-3}, \eta_{N} \approx 1.5$ accounts for nuclei heavier than hydrogen, in both CRs and in the target gas. For estimation of the $\mathrm{H}_{2}$ target mass $M$ based on the CS radio maps we used the procedure described by the authors in Sect. 4.2 of Tsuboi et al. (1999):

$$
\begin{aligned}
M\left(\mathrm{H}_{2}\right)\left[M_{\odot}\right]= & 7.5 \times 10^{11} T_{\mathrm{ex}}[\mathrm{K}] \\
& \times \frac{\int T_{\mathrm{MB}} \mathrm{d} v\left[\mathrm{~K} \mathrm{~km} \mathrm{~s}^{-1}\right] \cdot A\left[\mathrm{~cm}^{2}\right] \cdot \mu\left(\mathrm{H}_{2}\right)\left[M_{\odot}\right]}{X(\mathrm{CS})},
\end{aligned}
$$

where the excitation temperature $T_{\mathrm{ex}}$ of CS is $30 \mathrm{~K}, \int T_{\mathrm{MB}} \mathrm{d} v$ is the measured velocity-integrated antenna temperature, $A$ is the area of the GC region, $\mu\left(\mathrm{H}_{2}\right)$ is the mass of the hydrogen molecule and $X(\mathrm{CS})=10^{-8}$ is the relative abundance of $\mathrm{CS}$ in $\mathrm{H}_{2}$ clouds.

In the scan, we tested two different scenarios where: (1) the $\rho_{\mathrm{CR}} \propto r^{-\alpha}$ profile dominates the cosmic-ray density in the region and (2) the peaked cosmic-ray profile is found on top of an underlying homogeneous density, so that $\rho_{\mathrm{CR}} \propto r^{-\alpha}+$ const. To account for the background and telescope exposure uncertainties, we have generated 50 random exposure and background maps representing the uncertainty range of our reconstruction method and repeated the scan for each of them. We then averaged the resulting likelihood values in each bin of the scan phase space, which is equivalent to marginalization over these random map representations. The same technique of propagating the uncertainties of the background and exposure models has

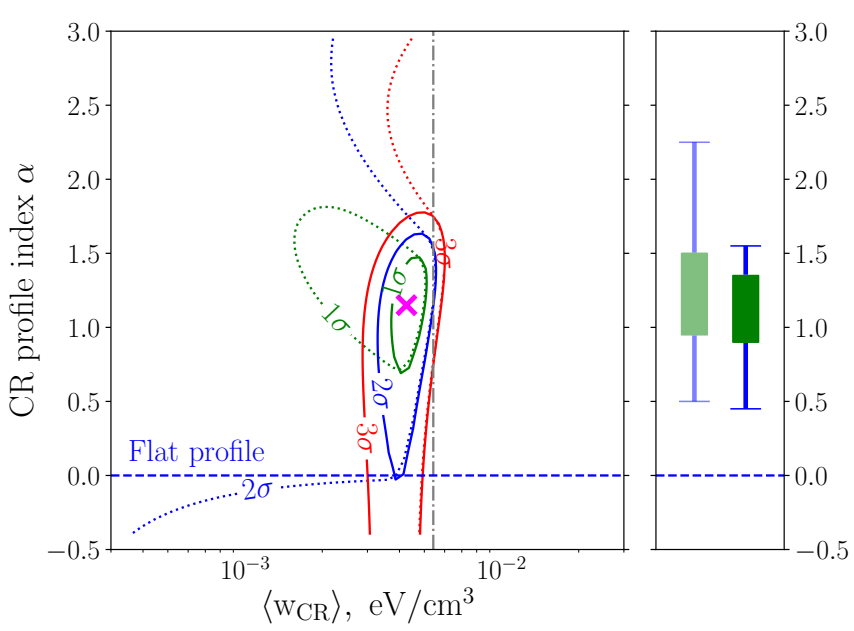

Fig. 3. Left: likelihood scan of the cosmic-ray density profile parameter space for $E_{\mathrm{CR}} \gtrsim 10 \mathrm{TeV}$, for a centrally peaked profile of the form $\rho_{\mathrm{CR}} \propto 1 / r^{\alpha}$, based on the MAGIC measurements. The scan has been performed for two different model assumptions: (1) only the cosmicray population with the power-law distribution exists (solid lines) and (2) the power-law population exist on top of a uniform cosmic-ray density distribution (dashed lines). Magenta crosses mark the best fit values for both assumptions, which nearly exactly overlap for our data. Gray vertical dash-dotted line represents the mean cosmic-ray density from H.E.S.S. Collaboration (2016). The mean cosmic-ray energy density $\left\langle w_{\mathrm{CR}}\right\rangle$ estimated here corresponds to the Galactic longitude range $\left[-1^{\circ} ; 1^{\circ}\right]$. Right: marginalization of the scan over the cosmic-ray profile index only. Green boxes and blue error bars mark the 1 and $2 \sigma$ confidence ranges correspondingly for assumptions 1 (dark colours) and 2 (light colours).

also been applied throughout the spectral analysis (Sect. 3.4). The resulting averaged values were then used to compute the confidence contours, shown in Fig. 3. In addition to $\alpha-w_{\mathrm{CR}}$ combined confidence contours, the right panel of the same figure shows the marginalized uncertainties for the power-law index $\alpha$ for both scenarios (1) and (2), with darker and lighter colours, respectively.

Scenario (1) favours a cosmic-ray density profile with $\alpha=$ $1.2_{-0.3}^{+0.2}(1 \sigma$ uncertainty). A similar profile with $\alpha=1.2 \pm 0.3$ is found for scenario (2), where a homogeneous contribution to the cosmic-ray profile is allowed.

As illustrated in Fig. 4, the full likelihood fit to the obtained sky map is also inconsistent with the $\rho_{\mathrm{CR}}=$ const assumption. To perform this fit, we first split the CS emission map of Tsuboi et al. (1999; integrated over the radial velocity) into a sequence of concentric rings, with their own normalization factors. Since the CS emission is highly peaked toward the Galactic plane, this is effectively equivalent to a longitudinal split of the Plane inside the $b=\left[-0.1^{\circ}, 0.1^{\circ}\right]$ stripe. When computing the normalizations of the rings, we've also included the Sgr A* point source, as well as G0.9+0.1 and the "Arc" source to the fitted model. The resulting cosmic-ray density profile, shown in Fig. 4 was ultimately fit with the $\rho_{\mathrm{CR}}=$ const model, yielding $\chi^{2} \approx 22$ over 5 degrees of freedom. This corresponds to $\approx 3.5 \sigma$ data to model disagreement, indicating a peaked profile.

In summary, MAGIC data above $1 \mathrm{TeV}$ indicate a radial cosmic-ray profile with power index $\alpha \approx 0.9-1.4$, which is somewhat different to, but still compatible with, earlier findings by H.E.S.S. Collaboration (2016). 


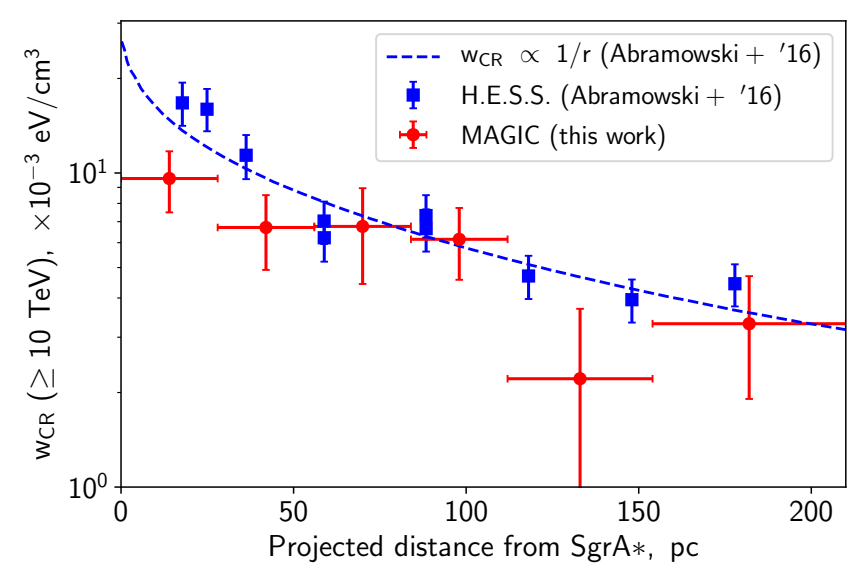

Fig. 4. Projected cosmic-ray energy density, as obtained from the full likelihood fit to the MAGIC sky map above $1 \mathrm{TeV}$. The projected distance is counted from the GC position. Measurements of H.E.S.S. Collaboration (2016) are shown in blue for comparison.

\subsection{Spectral analysis of the detected sources}

To compute the energy spectra of the detected sources in the MAGIC field of view - including the diffuse Galactic plane emission - we used the SkyPrism package. The spatial model used for the fit includes three point sources (Sgr A*, G0.9+0.1 and the Arc source at RA $=17: 46: 00, \mathrm{Dec}=-28: 53: 00)$ and the velocity-integrated CS map, re-scaled with the $\rho_{\mathrm{CR}} \propto r^{-1.2}$ bestfit cosmic-ray profile. The fit was performed in the energy range from $400 \mathrm{GeV}$ to $50 \mathrm{TeV}$ with two methods - first, based on energy bins (7 logarithmic energy bins) and second, assuming a certain spectral shape model for each of the sources. In the latter case, we applied a forward folding procedure considering the energy migration matrix during the fit. All spectra were generated from the general form

$\mathrm{d} N / \mathrm{d} E=N\left(\frac{E}{E_{0}}\right)^{\left(\Gamma+\beta \log \left(E / E_{0}\right)\right)} \exp \left(-E / E_{\text {cut }}\right)$,

which can result in a power-law, log-parabola, and power-law with cut-off spectral shape depending on the choice of $\beta$ and $E_{\text {cut }}$. The normalization energy for all the sources was set to $E_{0}=$ $2 \mathrm{TeV}$, keeping the correlation between the spectral parameters minimal.

To obtain the best fit parameters of the assumed spectral models, we performed a maximal Poissonian likelihood fit to the energy-binned MAGIC sky maps. To ensure a good level of accuracy for the estimated uncertainties, we additionally used the Markov chain Monte Carlo (MCMC) sampler emcee on the parameter space (Foreman-Mackey et al. 2013). The uncertainties of the exposure and background models derived from Monte Carlo simulations and data regions more than $0.3^{\circ}$ off the Galactic plane were propagated to the final results by processing 60 random representations through the MCMC sampler and merging the samples. The best-fit values for the detected sources, obtained through this fit, are given in Table 1, along with the corresponding errors and detection significance figures. The obtained spectra (data points and fit results) are shown in Fig. 5. The data points are not the result of spectral unfolding but, rather, spillover corrections based on the energy migration matrix and the fitted spectral shape were applied. The obtained MAGIC spectrum is consistent with the earlier estimate of the Galactic ridge spectral energy density (SED; H.E.S.S. Collaboration 2018), as displayed in Fig. 6. A likelihood ratio test comparing the model for the diffuse component with cut-off to a pure powerlaw results in the $\approx 2 \sigma$ preference for the cut-off for the MAGIC data set.

The SED shown earlier by H.E.S.S. Collaboration (2016) that led to the speculation about a possible $\mathrm{PeV}$ proton accelerator (PeVatron) at the GC, shows a lower average flux and somewhat different spectral shape compared to the other two SEDs in Fig. 6. This difference could be explained by the fact that also the regions in which the fluxes were measured are different. While H.E.S.S. Collaboration (2018) and this work try to include the whole $<1 \mathrm{deg}$ from the GC part of the Galactic ridge, avoiding point sorces, H.E.S.S. Collaboration (2016) used a donut-shaped region for extracting their flux, with a cut-out at the position of the Arc source, and inner and outer radii of $0.15 \mathrm{deg}$ and $0.45 \mathrm{deg}$, respectively.

We estimate the systematic uncertainties, arising from uncertainties on the energy and flux normalization scales, following the procedure discussed in Ahnen et al. (2017a; based on a detailed study by Aleksić et al. 2016). The resulting estimates are indicated by gray arrows in Figs. 5 and 6, where the vertical arrows indicate the effect of the flux normalization errors at different energies and the horizontal or inclined arrows indicate the effect of the energy scale uncertainty.

\section{Estimation of the possible biases in the analysis}

\subsection{Bias from the background modelling}

In order to quantify the bias resulting from the background estimation, we used a simplified simulation of the background map based on the initial assumption on the extension and brightness of the sources in the GC region. In this simulation, we assume that the true signal measured by MAGIC consists of five contributions: extended gas emission (assumed to be traced by the CS map Tsuboi et al. 1999), point-like Sgr A*, the "Arc" source (Archer et al. 2016; Ahnen et al. 2017a; H.E.S.S. Collaboration 2018), point-like G0.9+0.1, and isotropic background. The relative normalizations of these components are taken from the previous analysis of Ahnen et al. (2017a); we used our best fit results for a cross-check. This composite image is then used to sample the photons in the telescope camera coordinates as a function of pointing azimuth and zenith, following the MAGIC pointing during the GC observations. The resulting event list is supplied to the background estimation routine for a comparison of the reconstructed versus the assumed background.

Based on the results, illustrated in Fig. 7, we expect the bias to stay below $2 \%$ in units of background flux nearly everywhere in the sky-maps. This translates to a bias of the measured flux of the diffuse emission of more than $40 \%$ in some regions along the edges of the Galactic plane, but less than $30 \%$ for the brighter regions along the plane and at the center. Still, using the CS map as an approximation for the $\gamma$-ray emission, the total bias on the integral flux of the Galactic plane component is estimated to be in the range $7-12 \%$.

\subsection{Bias and uncertainty from the assumed gas distribution model}

An accurate modeling of the $\gamma$-ray emission from GC region requires detailed knowledge of the gas distribution in three dimensions. The angular resolution of MAGIC is better than $0.1^{\circ}$, which translates into $\approx 15 \mathrm{pc}$ at $8.5 \mathrm{kpc}$ distance from the Earth. As a result, MAGIC can map the profile of $\gamma$-ray emission at the projected distances of tens of parsecs from the 
Table 1. Spectral fit results of the sources, detected in the MAGIC field of view.

\begin{tabular}{lccccc}
\hline \hline Name & Spec. type & $N, 10^{-25} \mathrm{eV}^{-1} \mathrm{~cm}^{-2} \mathrm{~s}^{-1}$ & $\Gamma$ & $E_{\text {cut }}, \mathrm{TeV}$ & Detection significance \\
\hline Sgr A* & PLC & $5.39_{-0.46}^{+0.56}$ stat. $_{-1.19}^{+1.61}$ sys. & $-1.98_{-0.10}^{+0.11}$ stat. $_{-0.17}^{+0.18}$ sys. & $12.4_{-3.2}^{+5.5}$ stat. $_{-0.2}^{+3.3}$ sys. & $\approx 48 \sigma$ \\
G0.9+0.1 & PL & $0.93_{-0.17}^{+0.20}$ stat..$_{-0.12}^{+0.26}$ sys. & $-2.32_{-0.15}^{+0.13}$ stat. $_{-0.12}^{+0.20}$ sys. & - & $11.1 \sigma$ \\
Arc & PL & $0.52_{-0.15}^{+0.15}$ stat. $_{-0.09}^{+0.16}$ sys. & $-2.29_{-0.19}^{+0.17}$ stat. ${ }_{-0.13}^{+0.23}$ sys. & - & $6.4 \sigma$ \\
Diffuse & PLC & $9.32_{-1.63}^{+2.39}$ stat. $_{-1.97}^{+2.53}$ sys. & $-1.98_{-0.21}^{+0.26}$ stat. $_{-0.15}^{+0.16}$ sys. & $17.5_{-9.55}^{+59.3}$ stat. $_{-1.9}^{+4.5}$ sys. & $17.3 \sigma$ \\
\hline
\end{tabular}

Notes. The spectrum type acronyms stand for: PL - power-law and PLC - cut-off power-law. Normalization factor $N$ is given in units of $10^{-25}\left[\mathrm{ph} /\left(\mathrm{cm}^{2} \mathrm{~s} \mathrm{eV}\right)\right]$; normalization energy is set to $E_{0}=2 \mathrm{TeV}$ for all the sources. The curvature parameter $\beta=0$ in all cases. For the sources fitted with the power-law model the values of $E_{\text {cut }}$ are not given. All uncertainties correspond to a $68 \%$ confidence interval.

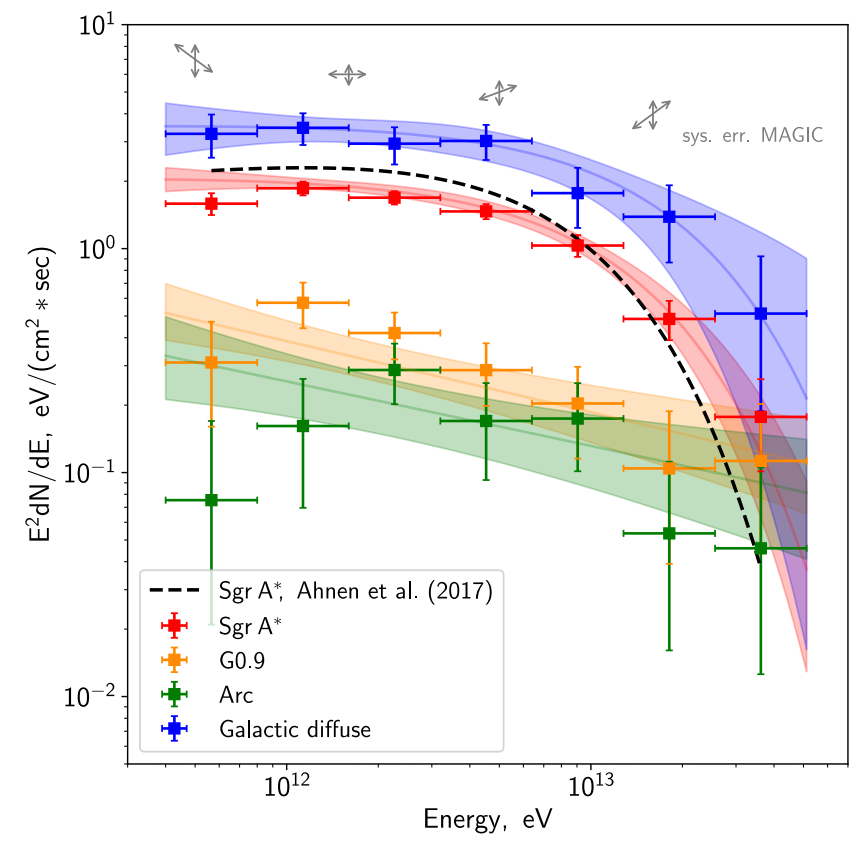

Fig. 5. MAGIC SEDs of the different components in our model, data points and forward folding fit results (colored bands). For the components corresponding to Sgr A*, the "Arc" and the CR/MC component a power-law shape with exponential cut-off has been used while G0.9+0.1 can be described with a simple power-law. The error bars and bands were computed from the MCMC samples and correspond to $68 \%$ confidence range. No spectral unfolding has been applied to the data points, but the effect of spillovers due to energy migration has been corrected for, based on the spectral shapes. Gray arrows indicate the size (length) and direction (orientation) of the SED shifts due to the systematical uncertainties in the analysis (see Sect. 3.4 for details).

position of the central supermassive black hole (SMBH); a conversion of this profile to the cosmic-ray density distribution naturally requires, then, that the line-of-sight distances to the gas clouds in the region are known with similar or better accuracy.

This requirement is very difficult to fulfill in practice. At larger distances the locations of the gas clouds are generally inferred from their kinematics, assuming a certain model of gas orbital motion (e.g., Sofue 1995; Nakanishi \& Sofue 2003. In the vicinity of the GC, however, this approach can no longer be applied, as the inability to put the source in front or behind the black hole image plane at the scales of tens of parsecs leads to degeneracy in the calculations. The required information - to a certain degree - can be reconstructed using measurements of line-of-sight absorption of the molecular cloud emission, which provides the necessary line-of-sight position

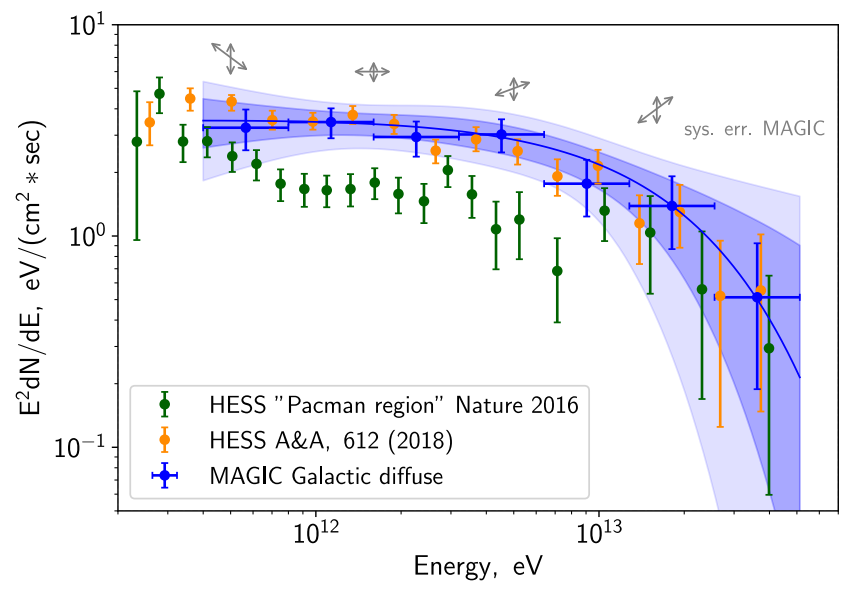

Fig. 6. Spectrum of the diffuse Galactic emission, derived from MAGIC data. Dark and light-blue regions mark the $68 \%$ and $95 \%$ confidence ranges for the assumed power-law with exponential cut-off model. The diffuse spectrum from H.E.S.S. Collaboration (2018), extracted from a similar region, is shown in orange, while the SED obtained from a cut annulus with $0.45 \mathrm{deg}$ outer radius from H.E.S.S. Collaboration (2016) is shown in green. Gray arrows indicate the possible shifts due to the systematical uncertainties in the analysis, similar to Fig. 5.

estimates (Sawada et al. 2004). Nevertheless, the line-of-sight locations of separate clouds in the GC region can hardly be reconstructed with an accuracy better than $\approx 50 \mathrm{pc}$.

In the absence and therefore negligence of the line-of-sight information, Eq. (1) naturally simplifies to Eq. (2), which works only with the projected gas and cosmic-ray densities. Depending on the real distribution $\rho_{\text {gas }}(x, y, z)$, the transition Eqs. (1) and (2) may bear an oversimplification, resulting in a biased cosmic-ray profile $\rho_{\mathrm{CR}}(r)$.

We do not attempt to reconstruct a fully realistic structure of the central $\approx 200$ pc of our Galaxy, which would be rather complex (Ferrière et al. 2007). Still, in order to quantify the associated bias in our analysis, we reconstruct the $3 \mathrm{D}$ gas distribution in the GC region based on the measurements of Sawada et al. (2004) and Tsuboi et al. (1999). This reconstruction then allows us to compare the profiles obtained accounting for or neglecting the line-of-sight information.

To perform the reconstruction, we used the fact that the $2 \mathrm{D}$ CS gas emission images of Tsuboi et al. (1999) are complemented by the radial velocity $v_{\text {rad }}$ information, which already provides the line-of-sight information in an indirect way. A mapping of $v_{\text {rad }}$ to the missing $z$ coordinate is found in Sawada et al. (2004), where it is given in the $(x, z)$ projection (in our notation). Hence, the measured intensity of the radio emission $I\left(x, y, v_{\mathrm{rad}}\right)$ 

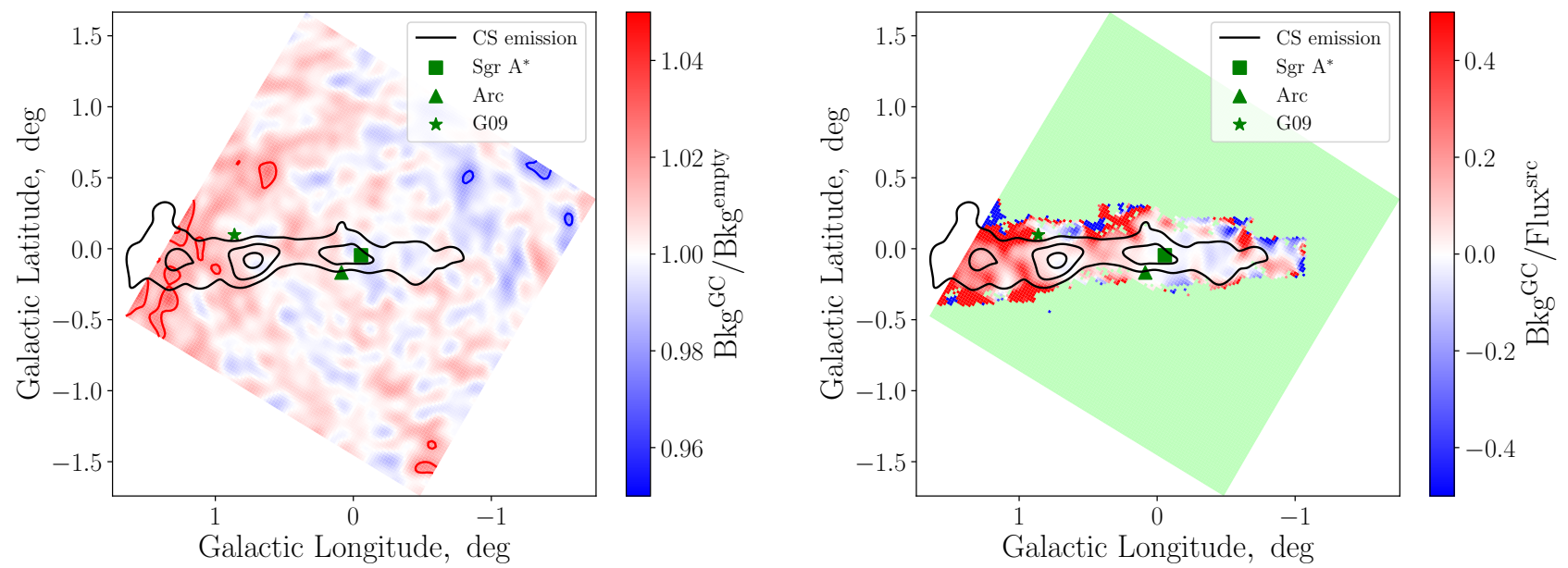

Fig. 7. Left: background bias (in units of the true assumed background flux Bkg ${ }^{\text {empty }}$ ) for the applied background reconstruction technique, estimated with simulations. The map was smeared with the Gaussian kernel with $\sigma=0.05^{\circ}$; contours indicate $\pm 2 \%$ differences with respect to the reference value of 1. Also plotted are the positions of the known $\gamma$-ray sources in the Galactic center region as well as the CS emission contours from Tsuboi et al. (1999), corresponding to the integral antenna temperatures of 5, 10 and $15 \mathrm{~K}$, smeared with the same Gaussian kernel. Right: same, but in units of the source flux for the assumed model. Here, only the uncertainties within the region of the used CS gas map are plotted, as the rest of the modelled image contains only background. Such background-only regions are filled with light green.

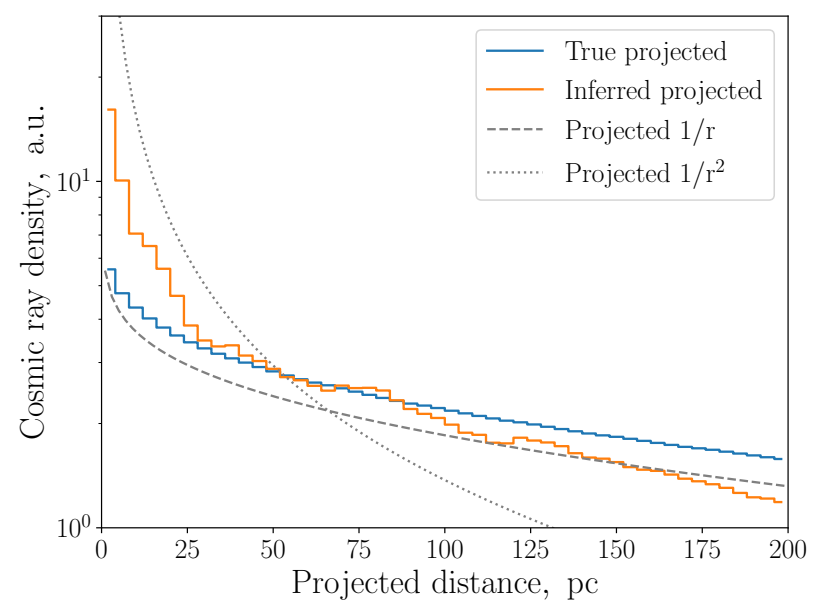

Fig. 8. Projected cosmic-ray density as a function of the distance from the GC, computed using a 3D reconstruction of the gas distribution in the region. The underlining profile is assumed to have a $\rho_{\mathrm{CR}} \propto r^{-1}$ shape. Blue line shows the true projection, whereas the orange one depicts the $P_{\mathrm{CR}}^{\prime}(r)=S(r) / P_{\text {gas }}(r)$ estimate. Dashed and dotted grey lines represent the exact solutions for the $\alpha=\{1,2\}$ cases respectively, arbitrarily scaled to fit the image. The calculation was performed for the \pm 10 pc Galactic latitude slice along the Galactic plane.

can be mapped to a full 3D cube $I(x, y, z)$ through a relation $v_{\text {rad }}(x, y) \leftrightarrow v_{\text {rad }}(x, z)$. The obtained cube gives an approximate picture of $3 \mathrm{D}$ gas distribution in the innermost $\approx 400 \times 400 \times$ $100 \mathrm{pc}$ of the GC region.

Despite the limitations of this approach, based on this $3 \mathrm{D}$ cube one can quantify the biases in the calculations from the previous section. In particular, we can check whether the transition Eqs. (1) and (2) still gives a valid estimate for a realistic gas density distribution.

To verify this, we took the example case of a $\rho_{\mathrm{CR}} \propto r^{-1}$ profile and computed the projected cosmic-ray density $P_{\mathrm{CR}}(r)$ directly and using the $P_{\mathrm{CR}}^{\prime}(r)=S(r) / P_{\text {gas }}(r)$ relation. The result of this comparison is shown in Fig. 8, where the exact projected shapes for $\alpha=\{1,2\}$ profiles are also given.

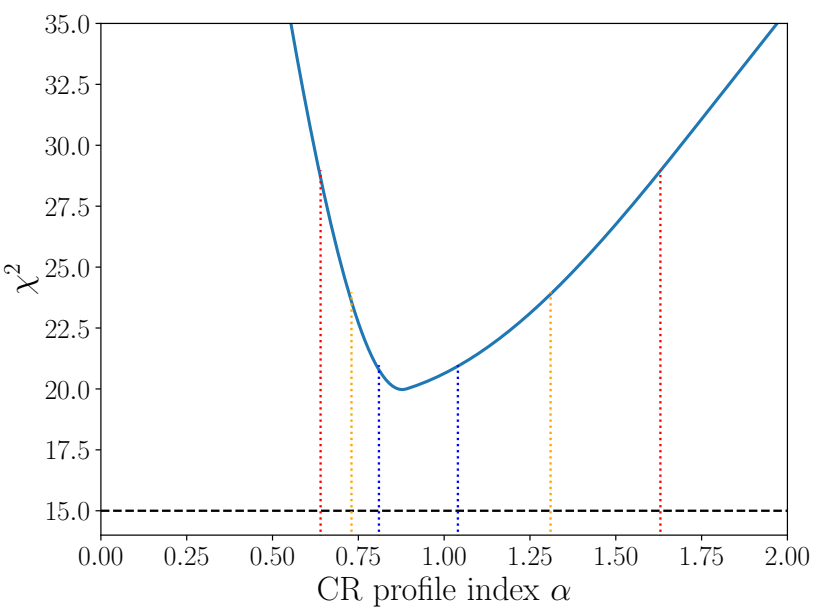

Fig. 9. Scan of the cosmic-ray density profile power-law index $\alpha$ with respect to the data in Fig. 4 and earlier measurements from H.E.S.S. Collaboration (2016). The vertical dashed lines mark the derived 1 (blue), 2 (orange) and $3 \sigma$ (red) confidence intervals. The horizontal dashed line represent the number of degrees of freedom in the fit. The scanned value, $\alpha$ corresponds to the true 3D cosmic-ray distribution and is projected on the plane of the sky using an assumed 3D gas map. See Sect. 4.2 for details.

It is clear from this figure that the inferred profile in the $\alpha=1$ case would resemble a steeper one with $\alpha^{\prime} \approx 1.5$. In this way, an $\alpha^{\prime} \approx 1.0-1.5$ measurement, presented in Sect. 3.3, is likely to correspond to the true $\alpha \approx 1$ distribution, accounting for this bias. In order to directly check this assumption, we performed a fit of inferred profiles of a form $\rho_{\mathrm{CR}} \propto r^{-\alpha}+$ const for $\alpha=[0 ; 2]$ to the projected cosmic-ray densities, obtained here (Fig. 4) and earlier in H.E.S.S. Collaboration (2016). The results of this test, shown in Fig. 9, indeed suggest that the true, de-projected cosmic-ray profile has a slope of $\alpha=0.88_{-0.07}^{+0.16}$ (at $68 \%$ confidence level), consistent with the $\alpha=1$ assumption.

In a similar way, we can also estimate the effect of the uncertain line-of-sight measurements on the derived value of $\alpha$. 


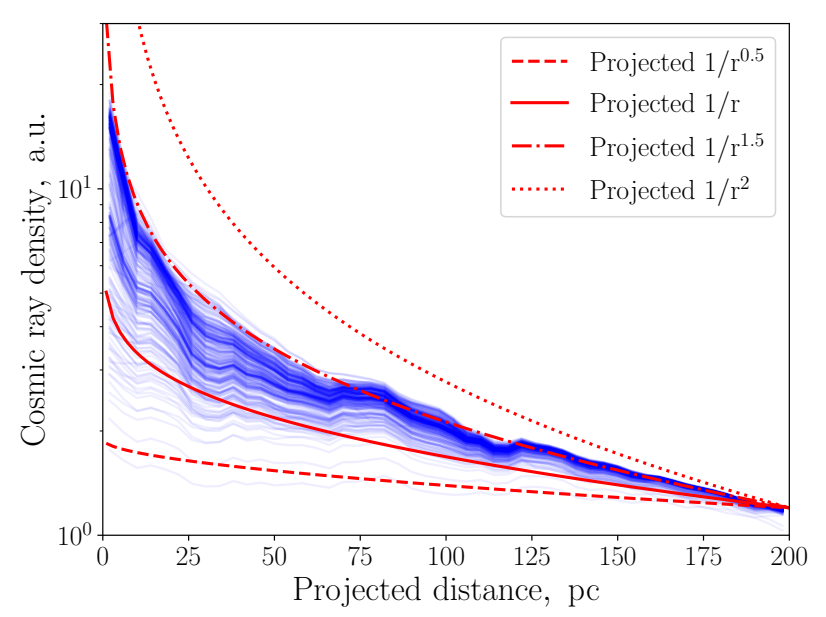

Fig. 10. Projected cosmic-ray density as a function of the distance from the GC, computed using a 3D reconstruction of the gas distribution in the region. The profiles are inferred from the $P_{\mathrm{CR}}^{\prime}(r)=S(r) / P_{\text {gas }}(r)$ estimate using the random line-of-sight shifts of the underlying gas distribution (see Sect. 4.2 for details). The true profile assumed in the simulation is $\rho_{\mathrm{CR}} \approx r^{-1}$. Dashed and dotted red lines represent the exact solutions for the $\alpha=\{0.5,1,1.5,2\}$ cases, respectively, arbitrarily scaled to fit the image.

To reconstruct the effect we randomly shifted the positions of the cube grid cells in the $z$ direction and then regenerated the expected cosmic-ray profiles $P_{\mathrm{CR}}^{\prime}(r)$. The random shifts were obtained by adding 10-40 Gaussian-distributed displacements with standard deviations from 50 to $200 \mathrm{pc}$ at random positions within the cube. The amplitude of the Gaussians was fixed to 25 parsec, while their width puts a coherence scale, not allowing the neighbouring bins to have very different shifts. The resulting shifts in the cube do not exceed $\approx \pm 50 \mathrm{pc}$, roughly resembling the corresponding measurement uncertainties.

The outcome of this calculation is shown in Fig. 10. The comparison with the exact $\alpha=\{1,2\}$ profiles projections indicates that the uncertainty in the line-of-sight position measurements can dramatically change the shape of the measured cosmic-ray profile. Within our simulation setup, assuming an $\alpha=1$ cosmic-ray profile, we can generally conclude that a measurement of $\alpha^{\prime} \approx 1.5$ is equally likely.

It should also be noted that the main evidence for the centrally peaked cosmic-ray profile comes from the measurements in the central $\approx 30-50 \mathrm{pc}$, where the uncertainty on the gas content is the highest. Any unaccounted absorption of the molecular radio emission from such dense regions may lead to a general underestimation of the gas content in the direct vicinity of the GC (in addition to the line-of-sight uncertainty) and thereby to a more modest estimate of the cosmic-ray density there.

\section{Discussion}

Based on deep VHE $\gamma$-ray observations using the MAGIC telescopes, we can reconstruct the cosmic-ray distribution profile in the $\mathrm{GC}$ region. In this process, we use the simplifications outlined in Sect. 3.3, which result from our partial knowledge of the line-of-sight distribution of the target gas material. This implies that measurements of the cosmic-ray distribution in the GC vicinity can only be improved with more accurate gas observation in other (radio, infra-red, etc.) domains and are not limited by the sensitivity of current $\gamma$-ray instruments. Accordingly, even highly sensitive future CTA observations of this region, for an analysis like the one presented here, depend on higher resolution molecular line measurements.

Our estimations, described in Sect. 4.2, indicate that in the absence of such measurements the derived cosmic-ray profile may look sharper than it is in reality. At the same time, the scatter in the profiles, based on the quoted uncertainties in the line-ofsight gas clouds positions, may make any interpretation of the measured shape less reliable. As an example, the $\alpha=1$ cosmicray profile, simulated in Sect. 4.2, may appear as $\alpha$ between 0.5 and 1.5 depending on the exact locations of the clouds (see Fig. 10).

The existence of a centrally peaked cosmic-ray distribution around GC, revealed by the H.E.S.S. data (H.E.S.S. Collaboration 2016) and confirmed here, however, seems reliable. The MAGIC data suggest an $\alpha=1.2 \pm 0.3$ profile and therefore do not contradict the $\alpha=1$ scenario, in which CRs diffuse outwards from a central source, especially given the considerations outlined above.

This peaked cosmic-ray profile, together with a hard emission spectrum, was interpreted as a signature of $\mathrm{PeV}$ cosmic-ray acceleration by SgrA* (H.E.S.S. Collaboration 2016). It should be noted here that alternative explanations for the enhanced density of CRs at GC exist, ranging from millisecond pulsars (Guépin et al. 2018) to dark matter (Lacroix et al. 2016). It has also been shown by Gaggero et al. (2017) that it is possible to consistently model the diffuse $\gamma$-ray emission from GC in the Fermi and VHE emission energy range with particles from the Galactic CR sea, nearly without the need of an addition central CR source. The authors also state that in the presence of such a CR sea, the maximum energy of any excess from a central source is even less certain.

MAGIC measurements of the diffuse $\gamma$-ray spectrum over a larger region of the central molecular zone, $\approx 150 \mathrm{pc}$ in width, favor, on $\mathrm{a} \approx 2 \sigma$ level, a cut-off in the $\gamma$-ray spectrum over a pure power law. The $1 \sigma$ confidence range for the cut-off energy spans from $10 \mathrm{TeV}$ to $80 \mathrm{TeV}$ (see Sect. 3.4). This corresponds to proton energies of $\approx 0.1-1 \mathrm{PeV}$, which means that the data are still marginally compatible with the PeVatron scenario.

\section{Conclusions}

The presence and proximity of a central super-massive black hole make the GC region a unique laboratory for studying comic-ray acceleration near black holes. The above-presented MAGIC observations confirm that the cosmic-ray density profile in the GC vicinity is peaked towards the position of the central SMBH of our Galaxy, similar to what has been pointed out by H.E.S.S. Collaboration (2016). This is consistent with the idea of activity of the latter as a particle accelerator in the recent past or, possibly, even nowadays. The MAGIC data also show that the spectrum of this diffuse $\gamma$-ray emission is hard $(\Gamma \approx 2)$ and reaches energies of several ten TeV. Our analysis, however, also reveals a hint of the presence of a spectral turnover at around those energies at the $2 \sigma$ level.

In order to gain further insight to particle acceleration and diffusion in the GC region, including the PeVatron topic, even deeper observations of the GC region by current and nextgeneration instruments are necessary. The focus should be on energies around and above $10 \mathrm{TeV}$, where current data sets are still statistics limited. Our study also indicates that such detailed measurements will be very sensitive to the line-of-sight gas distribution assumed and, as such, would require a deeper and higher resolution radio survey of the central $200 \mathrm{pc}$ of our Galaxy. 
Acknowledgements. We would like to thank the Instituto de Astrofísica de Canarias for the excellent working conditions at the Observatorio del Roque de los Muchachos in La Palma. We also thank Dario Grasso for the valuable comments. The financial support of the German BMBF and MPG, the Italian INFN and INAF, the Swiss National Fund SNF, the ERDF under the Spanish MINECO (FPA2015-69818-P, FPA2012-36668, FPA2015-68378-P, FPA2015 69210-C6-2-R, FPA2015-69210-C6-4-R, FPA2015-69210-C6-6-R, AYA201571042-P, AYA2016-76012-C3-1-P, ESP2015-71662-C2-2-P, FPA2017-90566REDC), the Indian Department of Atomic Energy, the Japanese JSPS and MEXT, the Bulgarian Ministry of Education and Science, National RI Roadmap Project DO1-153/28.08.2018 and the Academy of Finland grant nr. 320045 is gratefully acknowledged. This work was also supported by the Spanish Centro de Excelencia "Severo Ochoa" SEV-2016-0588 and SEV-2015-0548, an Unidad de Excelencia "María de Maeztu" MDM-2014-0369, by the Croatian Science Foundation (HrZZ) Project IP-2016-06-9782 and the University of Rijeka Project 13.12.1.3.02, by the DFG Collaborative Research Centers SFB823/C4 and SFB876/C3, the Polish National Research Center grant UMO2016/22/M/ST9/00382 and by the Brazilian MCTIC, CNPq and FAPERJ.

\section{References}

Abdo, A. A., Ackermann, M., Ajello, M., et al. 2010, Phys. Rev. Lett., 104, 101101

Aharonian, F., Akhperjanian, A. G., Bazer-Bachi, A. R., et al. 2006a, ApJ, 636, 777

Aharonian, F., Akhperjanian, A. G., Bazer-Bachi, A. R., et al. 2006b, Nature, 439, 695

Ahnen, M. L., Ansoldi, S., Antonelli, L. A., et al. 2017a, A\&A, 601, A33

Ahnen, M. L., Ansoldi, S., Antonelli, L. A., et al. 2017b, Astropart. Phys., 94, 29

Albert, J., Aliu, E., Anderhub, H., et al. 2008, Nucl. Instrum. Meth. Phys. Res. A, 588, 424

Aleksić, J., Ansoldi, S., Antonelli, L. A., et al. 2016, Astropart. Phys., 72, 76

Archer, A., Benbow, W., Bird, R., et al. 2016, ApJ, 821, 129

Ferrière, K., Gillard, W., \& Jean, P. 2007, A\&A, 467, 611

Fomin, V. P., Stepanian, A. A., Lamb, R. C., et al. 1994, Astropart. Phys., 2, 137

Foreman-Mackey, D., Hogg, D. W., Lang, D., \& Goodman, J. 2013, PASP, 125, 306

Fruck, C., Gaug, M., Zanin, R., et al. 2014, ArXiv e-prints [arXiv:1403. 3591]

Gaggero, D., Grasso, D., Marinelli, A., Taoso, M., \& Urbano, A. 2017, Phys. Rev. Lett., 119, 031101

Gaug, M., Blanch, O., Dorner, D., et al. 2014, ArXiv e-prints [arXiv:1403. 5083]

Guépin, C., Rinchiuso, L., Kotera, K., et al. 2018, JCAP, 2018, 042

H.E.S.S. Collaboration (Aharonian, F. A.) 2006, Nature, 439, 695

H.E.S.S. Collaboration (Abramowski, A., et al.) 2016, Nature, 531, 476

H.E.S.S. Collaboration (Abdalla, H., et al.) 2018, A\&A, 612, A9

Hunter, S. D., Bertsch, D. L., Catelli, J. R., et al. 1997, ApJ, 481, 205

Lacroix, T., Silk, J., Moulin, E., \& Bœhm, C. 2016, Phys. Rev. D, 94, 123008

Nakanishi, H., \& Sofue, Y. 2003, PASJ, 55, 191

Oka, T., Hasegawa, T., Sato, F., Tsuboi, M., \& Miyazaki, A. 1998, ApJS, 118 455

Sawada, T., Hasegawa, T., Handa, T., \& Cohen, R. J. 2004, MNRAS, 349, 1167 Sofue, Y. 1995, PASJ, 47, 527

Tsuboi, M., Handa, T., \& Ukita, N. 1999, ApJS, 120, 1

van Eldik, C. 2015, Astropart. Phys., 71, 45

Vovk, I., Strzys, M., \& Fruck, C. 2018, A\&A, 619, A7

Zanin, R., Carmona, E., Sitarek, J., Colin, P., \& Frantzen, K. 2013, Proc. of the 33st International Cosmic Ray Conference, Rio de Janeiro, Brasil
${ }^{1}$ Inst. de Astrofísica de Canarias, 38200 La Laguna, Spain

${ }^{2}$ Universidad de La Laguna, Dpto. Astrofísica, 38206 La Laguna, Tenerife, Spain

3 Università di Udine and INFN Trieste, 33100 Udine, Italy

${ }^{4}$ National Institute for Astrophysics (INAF), 00136 Rome, Italy

5 ETH Zurich, 8093 Zurich, Switzerland

6 Technische Universität Dortmund, 44221 Dortmund, Germany

7 Croatian Consortium: University of Rijeka, Department of Physics, 51000 Rijeka; University of Split - FESB, 21000 Split; University of Zagreb - FER, 10000 Zagreb; University of Osijek, 31000 Osijek; Rudjer Boskovic Institute, 10000 Zagreb, Croatia

${ }^{8}$ Saha Institute of Nuclear Physics, HBNI, 1/AF Bidhannagar, Salt Lake, Sector-1, Kolkata 700064, India

9 Centro Brasileiro de Pesquisas Físicas (CBPF), 22290-180 URCA, Rio de Janeiro, RJ, Brasil

10 IPARCOS Institute and EMFTEL Department, Universidad Complutense de Madrid, 28040 Madrid, Spain

11 University of Łódź, Department of Astrophysics, 90236 Łódź, Poland

12 Università di Siena and INFN Pisa, 53100 Siena, Italy

13 Deutsches Elektronen-Synchrotron (DESY), 15738 Zeuthen, Germany

14 Istituto Nazionale Fisica Nucleare (INFN), 00044 Frascati, Roma, Italy

15 Max-Planck-Institut für Physik, 80805 München, Germany

16 Institut de Física d'Altes Energies (IFAE), The Barcelona Institute of Science and Technology (BIST), 08193 Bellaterra, Barcelona, Spain

17 Università di Padova and INFN, 35131 Padova, Italy

18 Università di Pisa and INFN Pisa, 56126 Pisa, Italy

19 ICRANet-Armenia at NAS RA, 0019 Yerevan, Armenia

20 Centro de Investigaciones Energéticas, Medioambientales y Tecnológicas, 28040 Madrid, Spain

21 Universität Würzburg, 97074 Würzburg, Germany

22 Finnish MAGIC Consortium: Finnish Centre of Astronomy with ESO (FINCA), University of Turku, 20014 Turku, Finland; Astronomy Research Unit, University of Oulu, 90014 Oulu, Finland

23 Departament de Física and CERES-IEEC, Universitat Autònoma de Barcelona, 08193 Bellaterra, Spain

24 Japanese MAGIC Consortium: ICRR, The University of Tokyo, 277-8582 Chiba, Japan; Department of Physics, Kyoto University, 606-8502 Kyoto, Japan; Tokai University, 259-1292 Kanagawa, Japan; RIKEN, 351-0198 Saitama, Japan

25 Inst. for Nucl. Research and Nucl. Energy, Bulgarian Academy of Sciences, 1784 Sofia, Bulgaria

26 Universitat de Barcelona, ICCUB, IEEC-UB, 08028 Barcelona, Spain

27 Port d'Informació Científica (PIC), 08193 Bellaterra, Barcelona, Spain

28 Dipartimento di Fisica, Università di Trieste, 34127 Trieste, Italy

${ }^{29}$ INAF-Trieste and Dept. of Physics \& Astronomy, University of Bologna, Bologna, Italy 